\title{
La biblioteca como editora de contenidos
}

\section{MEI}

II, vol. 6 no 11

\author{
J ulio Alonso Arévalo ${ }^{1}$ \\ Marta Vázquez Vázquez ${ }^{2}$
}

Recibido el 19-06-2015

\section{Resumen}

Una de las características más innovadoras de la biblioteca del siglo 21 tiene que ver con la toma de una postura activa frente a la gestión y generación de contenidos. Con la llegada de la Web 2.0 las bibliotecas no sólo siguen salvaguardando y difundiendo información como han venido realizando a lo largo de su historia, también cada vez con más frecuencia crean nueva información con el objetivo de prestar los mejores servicios a sus ciudadanos, a través de recursos y servicios tales como la elaboración guías de investigación, boletines de alerta y novedades, recursos web, información a través de sus blogs, y como administradores de contenidos a través de repositorios y revistas de acceso abierto. Un paso más allá en esta dinámica tienen que ver con la biblioteca como editora y distribuidora de libros, especialmente en el ámbito local, siendo la impulsora, formadora, dinamizador y difusoras de las obras de los autores de su comunidad.

\section{Palabras clave}

bibliotecas, espacio, diseño, futuro, información a la comunidad, editoriales, edición, autoedición, libros electrónicos.

\section{Title}

The library as content editor

\begin{abstract}
One of the most innovative features of the library of the 21st century has to do with taking an active stance regarding management and content generation. With the advent of Web 2.0 libraries not only continue safeguarding and disseminating information as they have been doing throughout theirs history, they also more and more often create new information with the aim of providing the best services to the citizens, through resources and services such as the development of research guides, newsletters and news alerts, web resources, through their blogs, and as curators of content through repositories and open access journals. A further step in this dynamic has to do with considering the library as publisher and distributor of books, especially at a local level, being the prime mover, trainer, facilitator and broadcasters of works of the authors in their community.
\end{abstract}

\section{Keywords}

libraries, space, design, future, community information, publishing, editing, desktop publishing, electronic books. 


\section{Introducción}

Con la llegada de los recursos digitales a las bibliotecas van quedando atrás los días en que las bibliotecas eran puramente salas de lectura. Hoy en día las bibliotecas se están transformando en espacios versátiles, espacios polivalentes, que además de ofrecer todavía a la gente la oportunidad de acceder a una gran cantidad de materiales físicos de lectura, también proporcionan acceso a Internet, a dispositivos digitales, apoyo a las personas en la búsqueda de trabajo y aplicaciones móviles para facilitar el acceso a los recursos en línea; además de ofrecer oportunidades de aprendizaje en contextos informales, tales como espacios para reuniones y encuentros para el público en general, para grupos comunitarios y otras organizaciones locales.

En el momento actual casi todas las bibliotecas de Estados Unidos tienen un catálogo digital que ofrece libros electrónicos, La oferta de recursos digitales siguen aumentando, con más del $90 \%$ de las bibliotecas públicas que ofrecen ebooks. Una reciente encuesta de OverDrive, el mayor proveedor de libros electrónicos para bibliotecas encontró que se prestaron más de 120 millones de libros electrónicos y audiolibros en los nueve primeros nueve meses de 2015, lo que representa año tras año un crecimiento medio de casi el $20 \%$. (2015d)

Pero además de sus colecciones de grandes distribuidores las bibliotecas están apostando firmemente y ofreciendo muchos títulos de autopublicación, especialmente de autores locales. Está es una de las grandes líneas de crecimiento de la colección digital en las bibliotecas de Estados Unidos, prácticamente todas bibliotecas y consorcios disponen de este tipo de obras. Por ello las bibliotecas públicas están mostrando cada vez más interés en el mundo editorial, no tanto como compradoras de contenidos sino como productoras o autoras de los mismos. Bajo la premisa de que si varias organizaciones sin experiencia en el mundo editorial se están convirtiendo en editores, ¿Porqué las bibliotecas no podrían hacer lo mismo con la experiencia acumulada en torno al mercado editorial?

En este documento hacemos una análisis de cómo las incorporación de recursos electrónicos a las bibliotecas están sirviendo como catalizador para la concreción de algunos de los servicios que muchas bibliotecas venían anunciando en los últimos años, tales como los servicios de información a la comunidad, y como también están facilitando servicios de carácter innovador. Es el caso de los servicios relacionados con la biblioteca como creadora y promotora de contenidos, tanto propios como ajenos. Desde hace algunos años las bibliotecas realizan boletines de sumarios, boletines de novedades, guías de lectura, blogs, e incluso las bibliotecas universitarias están gestionando repositorios y revistas electrónicas en acceso abierto; una nueva función es la 
de la biblioteca como impulsora y editora de libros. Por ello analizamos algunas de las iniciativas que se están planteando en otros países en relación con esta cuestión, para finalmente ver la viabilidad de este tipo de proyectos.

\section{La biblioteca como editora de contenidos}

Hace unos 20 años Brewster Kahle dijo que "todo el mundo quiere ser un editor.” A esta situación añadimos ahora la publicación de biblioteca, una zona de cultivo de mucho interés para muchos (Kahle 1993). En junio de 2013, Jaime LaRue, director de la biblioteca del condado de Douglas, planteo en el suplemento digital de la ALA, «E-Conten», que las bibliotecas consideraran su potencial como futuras editoras de contenido en el ámbito local. "Hay varias razones por las cuales las bibliotecas públicas podrían querer avanzar en esta dirección", escribió LaRue: "Una vez que una biblioteca invierte en la infraestructura para gestionar libros electrónicos directamente de los editores, dispone de la misma infraestructura que le permite ser editor." (LaRue 2013). Así antes de que una biblioteca pueda adoptar este nuevo papel, se debe en primer lugar tendrá que:

- Establecer una infraestructura técnica.

- Construir nuevos sistemas de relaciones con editores, adquisiciones y flujos de trabajo

- Gestionar la demanda

Según LaRue, Imagínese este banner en el sitio web de la biblioteca: “Quieres escribir un libro? A continuación, la biblioteca proporciona una hoja de ruta para la escritura. La hoja de ruta, incluye listas de grupos de escritores locales, También incluiría listas, tasas y una clasificación de los editores locales, además tendría información sobre el diseño de la cubierta (y directorios de los diseñadores y sus tasas) y los horarios de los talleres, eventos de autor, y conferencias. Al final cuando el autor termine su libro: La biblioteca que le ayudó a escribirlo, le ayudará también a hacer la revisión final. La biblioteca le ayudará a proporcionar al autor información sobre un cierto nivel de protección de copia para el archivo. Le asesorará sobre cuestiones de copyright. La biblioteca mostrará su obra y la hará accesible a la comunidad local. La biblioteca va a comprar copias múltiples basadas en la demanda del título. La biblioteca hará posible su descubrimiento para que otros puedan comprar la obra desde el catálogo de la biblioteca. La biblioteca hará posible su 
descubrimiento para que otros puedan comprar la obra desde el catálogo de la biblioteca. ¿Y por qué iba a la biblioteca de hacer todo esto? Para esta razón LaRue dice:

"Hay una vieja broma: ¿Por qué la gente roba bancos? Conclusión: porque ahi es donde está el dinero. ¿Por la biblioteca ha de ayudar a escribir y promocionar libros de sus autores? Respuesta: porque ahi es donde los lectores son."

Las bibliotecas de Colorado ya ha puesto en marcha este proyecto a través del documento "Colorado's eBook Manifesto (Consortium 2013) un documento para orientar a las bibliotecas sobre políticas profesionales y editoriales en torno al libro electrónico. El consorcio a través de el proyecto Evoke http:// evoke.cvlsites.org/ están contactando y creando una lista de editores que están dispuestos a vender libros electrónicos directamente a las bibliotecas, que a su vez también están contactando con otros proyectos similares como los de la Red de bibliotecas de Marmot y el consorcio de las bibliotecas del Condado de Douglas (2013d). La idea de estas bibliotecas es aprender a manejar varios canales emergentes de contenido digital. Para ello han habilitado una web con todas las herramientas y ayudas que van desarrollando para que sirvan de marco de referencia a otras bibliotecas y consorcios que se ofrece gratuitamente a la comunidad bibliotecaria:

- Lista de socios editores que venden directamente a bibliotecas http:// evoke.cvlsites.org/files/2013/05/DCL-eBook-Publisher-ContactList.pdf (Libraries 2013)

- Arquitectura de información de código fuente abierto para desarrollar una plataforma propia

- Una sección dedicada a descubrir a los clásicos del Consorcio de Bibliotecas de Colorado, desde donde cualquier biblioteca pueden importar registros MARC de más de 500 títulos de libros electrónicos de "clásicos descargables" del Proyecto Gutenberg a través del catálogo de la biblioteca. http://www.clicweb.org/e-discover-home

- Marco jurídico y aspectos legales en torno al libro electrónico

- Lista trimestral comparativa de de precios del libro electrónico Douglas County Libraries Report http://evoke.cvlsites.org/category/ douglas-county-libraries/ 
Por otra parte lado, también supondría participar en los beneficios de este tipo de compras, y obtener en torno al 10\% del beneficio de la venta: de hecho esto es lo que ocurre cuando alguien desde el catálogo de OverDrive realiza una compra de un libro electrónico a través del botón "BUY IT NOW” (¡Cómprelo ahora!); pero también porque puede ayudar a resolver un par de inconvenientes actuales del préstamo bibliotecario digital. Hoy en día, los usuarios de bibliotecas deben esperar largas listas para que los libros electrónicos más populares estén disponibles. Pero ¿qué pasa si alguien no quiere esperar o quiere tener el libro electrónico que ha leído en préstamo de forma permanente? Si las bibliotecas tienen la capacidad de vender libros digitales, los clientes podrían simplemente haga clic en un botón para adquirir el título en el que tienen un alto interés. Permitir que la gente compre libros digitales a través del catálogo de la biblioteca pública debe ser posible con un poco desarrollo de software y algunos acuerdos con algunos editores, especialmente con librerías y editores locales independientes. En este momento, estos centros de barrio ofrecen acceso a todas las formas de medios de comunicación, sin costo adicional a los individuos. Con la posibilidad de comprar libros digitales en las bibliotecas cada ciudad puede tener una vibrante, agradable y cálida librería. Con un beneficio común para el autor y para la biblioteca. En este línea, algunas bibliotecas han comenzado a hospedar tecnologías de impresión bajo demanda (como la Espresso Book Machin) y talleres de escritura creativa, tratamiento de textos, formatos y sistemas de autopublicación.

Desde esta perspectiva la publicación de la biblioteca implicaría un conjunto diverso y creciente de actividades, que puede proporcionar servicios útiles para una amplia gama de investigadores y estudiantes. Aunque la definición de "publicación" no siempre está clara y, a menudo parece significar "difusión" en este contexto. Las bibliotecas apoyan todo tipo de programas. Sobre todo con revistas de acceso libre, y algunas ya editan libros. En estos casos la actividad es financiada en un número de maneras, pero los fondos operativos de la biblioteca general, son una parte importante de la ecuación; y por supuesto algunas bibliotecas expresan una misión de "recuperar la publicación" de las entidades comerciales rapaces. Para Kate Lara hay muchas oportunidades para la innovación por parte de los bibliotecarios y editores; y de hecho el $72 \%$ de las bibliotecas destinan recursos para el acceso abierto, aunque muchos estiman que los mismo sólo representan entre el 1-5\% de los recursos totales. (2015b) En la actualidad, la responsabilidad de los cargos de financiación para procesamiento de artículo (APC) recae fundamentalmente sobre el autor o la institución. El $70 \%$ de las bibliotecas están proporcionando financiación con cargo a su presupuesto. Sin embargo, se estima que esta equivale a menos del $1 \%$ del presupuesto para las suscripciones de recursos tradicionales. Sólo el 19\% de las instituciones involucradas en la financiación tiene un límite máximo establecido, por lo general esta financiación va desde los 2000 a 3,000\$. La 
mayoría de los encuestados en Open Access Library Survey: An investigation of the role of libraries in open access funding and support within institutions consideró que la biblioteca debería apostar activamente por el acceso abierto. Sin embargo, la participación en la financiación es un tema dado a la división de opiniones. Mientras algunos creen que la responsabilidad financiera debe recaer únicamente en el autor, otros consideraron que la biblioteca debe desempeñar un papel central en la financiación del acceso abierto, en parte, mediante el control de los fondos de APC. Se desprende de este estudio que aún no hay un camino claro hacia el futuro de la financiación del acceso abierto, pero se vislumbran muchas oportunidades para la innovación por parte de las bibliotecas y editores. (Lara 2014)

En el momento actual casi todas las bibliotecas de Estados Unidos tienen un catálogo digital que ofrece libros electrónicos, en las que además de sus colecciones de grandes distribuidores están apostando firmemente y ofreciendo muchos títulos de autopublicación, especialmente de autores locales (LibraryJournal 2014). Está es una de las grandes líneas de crecimiento de la colección digital en las bibliotecas de Estados Unidos, prácticamente todas bibliotecas y consorcios disponen de este tipo de obras. En septiembre pasado la Douglas County Libraries (DCL) anunció la creación de The Wire: A Writer's Resource (2013b), un programa impulsado por la biblioteca que ofrece información y formación a los aspirantes a autores que quieren escribir, publicar y encontrar mercados para sus libros.(Alonso-Arévalo, Cordón-García y Gómez Díaz 2014)

En 2011, las Bibliotecas del Condado de Douglas (el tercer sistema de bibliotecas más grande de Colorado) lanzó una nueva plataforma de libros electrónicos autogestionada que, en sus primeros 12 meses de operación, tuvo el apoyo de 10 pequeños y medianos editores. En junio de 2011, las Bibliotecas del Condado de Douglas (DCL) proporcionaban acceso a más de 7.000 títulos de libros electrónicos para sus siete bibliotecas, que dan servicio a cerca de 300.000 usuarios. En marzo de 2012, se lanzó una versión actualizada de la plataforma con la integración de títulos de Overdrive en alquiler, y en septiembre de 2013, la colección de libros electrónicos alojados en la plataforma de DCL alcanzó 35.000 (incluyendo 10.000 títulos de autoedición, tras llegar a un acuerdo con la editorial Smashwords). En un contexto donde los títulos autoeditados representan el segmento de más rápido crecimiento de los contenidos publicados, se prevé que aumente de los 350.000 nuevos títulos por año que tuvo en 2011 hasta los 600.000 nuevos títulos que tendrá el año 2015. Con la excepción del contenido licenciado y accesible a través de Overdrive y 3M, los títulos alojados en la plataforma de DCL son propiedad de la biblioteca; de esta manera las bibliotecas de DCL poseen en propiedad la copia del archivo digital y, por lo tanto, pueden garantizar el acceso perpetuo al 
mismo. En la compra de nuevos libros electrónicos de las editoriales participantes, DCL busca aplicar un enfoque similar al que anteriormente se aplicaba a los libros físicos, con la típica lista de precios. (Alonso-Arévalo et al. 2014b)

Las motivaciones que impulsaron a DCL a desarrollar su propia plataforma de libros electrónicos surgieron por multitud de factores:

- Una creciente demanda de los usuarios de la biblioteca para tener acceso a una mayor gama de títulos.

- Las importantes restricciones de acceso a sus títulos destinados al préstamo en bibliotecas que los 6 grandes editores estaban imponiendo.

- El dominio en el mercado impuesto por agregadores como Overdrive. DCL buscaba atenuar los costes de contenido digital a través de sus sistemas de bibliotecas, después de una política editorial que aumentó los precios de los libros electrónicos con incrementos de casi el $700 \%$ en un período de tres años

- La propensión de los agregadores, que incluye a Overdrive, 3M y Baker \& Taylor, a aumentar unilateralmente los precios, limitando el acceso de la biblioteca a contenido que previamente habían adquirido bajo licencia.

Con el fin de agilizar y facilitar las negociaciones con los editores para adquirir títulos digitales adicionales, DCL ha creado una carta estándar para producir una declaración de entendimiento común con los editores para la compra y adquisición de contenido digital.

Otro avance hacia la autogestión de plataformas y contenidos es el desarrollado por DCL y Colorado Library Consortium a través de su proyecto eVoke 2.0: Colorado Statewide eBook Pilot Project (2013c). El objetivo de este es desarrollar una infraestructura de contenidos digitales en la nube que proveerá capacidades de compra y préstamo de contenidos electrónicos a las bibliotecas de Colorado. Además también está desarrollando una plataforma basada en software libre que puede ser de utilidad para otras bibliotecas. Aunque es importante señalar que mientras el software de fuente abierta VuFind, que gestiona la capa de descubrimiento DCL es de libre uso, el proceso de ajustar adaptar y añadir funcionalidades adicionales a este software implica un coste. En junio de 2013, DCL llegó a un acuerdo con un proveedor de sistema para préstamo de ebook con sede en España, OdiloTID, para trabajar en asociación y para desarrollar una versión mejorada del modelo de DCL. A pesar de los considerables costes involucrados en el desarrollo de la plataforma de DCL, el modelo ha adquirido un impulso significativo en toda la comunidad 
bibliotecaria estadounidense, como en Marmot, la Red de Bibliotecas de Colorado, Arizona y en Carolina del Norte donde estas bibliotecas y consorcios están aplicando un modelo propio similar al de Douglas.

En mayo de 2013, CALIFA, la mayor Red de Bibliotecas de California (2015a), anunció el lanzamiento de su plataforma de libros electrónicos «Enki» de código abierto, que también se basa en el modelo de DCL, después de las malas experiencias con Overdrive debido a la no portabilidad de contenidos, y a las cláusulas impuestas por este agregador. La plataforma Enki utiliza una versión modificada de software de código abierto (VuFind +) como su capa de descubrimiento y el software «Adobe Content Server» para almacenar metadatos y manejar los libros con DRM. Enki facilita la adquisición colectiva e individual de la compra y alojamiento de títulos digitales de autores publicados, de pequeños editores y distribuidores independientes; lo que permite participar a las bibliotecas públicas más pequeñas en la compra directa y gestión de una parte significativa de sus colecciones de libros electrónicos sin una licencia sobre este contenido desde un agregador principal. Los objetivos del desarrollo de la plataforma Enki incluyen apoyo a la capacidad de las bibliotecas para poseer la licencia de compra de ebooks, y reducir el coste pagado por la misma con la finalidad de disponer de contenido por medio de compras colectivas, reduciendo de este modo la dependencia de los agregadores tales como $3 \mathrm{M}$ y Overdrive. Fomentan, además, un mayor descubrimiento y circulación de títulos de las editoriales independientes que trabajan con el modelo de autopublicación. El objetivo general es desarrollar una plataforma para la participación de las bibliotecas, cuyos contenidos sean perdurables a perpetuidad, tanto en el acceso como en la propiedad de los documentos digitales adquiridos.

La plataforma Enki actualmente alberga 18.000 títulos de más de 50 editoriales participantes, y está abierta a todas las bibliotecas públicas de California. La biblioteca Estatal de Kansas ofrece la plataforma Enki a todas las bibliotecas de Kansas después de contribuir a sus costes iníciales de desarrollo (2015c).

Por otra parte el Consorcio de Bibliotecas de Carolina del Norte, está experimentando con un nuevo modelo de negocio con libros electrónicos que proporciona a los usuarios de la biblioteca acceso ilimitado a más de 1.200 títulos de libros electrónicos de las editoriales con sede en Carolina del Norte; para ello el consorcio de bibliotecas se asoció con ocho casas editoriales locales para la compra de los libros electrónicos. Esta colección ofrece una amplia gama de contenido, incluyendo novelas de autores populares en Carolina del Norte, poesía, cuentos, y libros de no ficción. Los libros electrónicos están disponibles para el uso público en la plataforma BiblioBoard bajo la etiqueta de 
Medios Digitales. A diferencia de los libros electrónicos de la biblioteca tradicional, esta colección ofrece acceso siempre disponible y sin límite de usuarios simultáneos, es decir, los clientes no tendrán que hacer una reserva o esperar a que un libro electrónico esté disponible. Además, la plataforma BiblioBoard, editorial que sigue los preceptos de First Reader, permite al usuario visualizar los libros electrónicos en un navegador web o descargar en sus dispositivos, tales como una tableta a través de la aplicación BiblioBoard. El programa piloto se extenderá hasta diciembre de 2014, tiempo durante el cual NC LIVE trabajará con editores, bibliotecas y BiblioLabs, el proveedor de la plataforma BiblioBoard, para evaluar la sostenibilidad a largo plazo y el éxito de este modelo.(Corroto 2015)

Buscando la visibilidad de los autores independientes Biblioboard y Library Journal anunciaron en 2014 un proyecto completamente innovador. Se trata del lanzamiento de Self-e un servicio para ayudar a los bibliotecarios a identificar las obras digitales de autores independientes. Y no solo eso. Los escritores pueden proponer sus obras a través de este portal, con el compromiso por parte de Library Journal de analizar su viabilidad para las bibliotecas (estudio de formatos, contenido, etc.) e integrarlas en los catálogos en el caso de que sean aprobadas. Este servicio es gratuito para los autores que disfrutan de una licencia de no exclusividad.

(Polanka 2012)

Como mencionamos anteriormente, los libros autopublicaodos son una de las líneas de crecimiento de las colecciones en las bibliotecas públicas, especialmente en lo que se refiere a autores locales. Los autores eligen distribuir sus libros electrónicos a través de bibliotecas tal como lo harían con cualquier otro socio minorista, con la ventaja de que la biblioteca es un patrón de visibilidad importante. SELF-e es un proyecto colaborativo entre Library Journal y la plataforma de distribución de libros electrónicos BiblioBoard para apoyar a autores que se quieren autopublicar y poner sus libros en las bibliotecas con acceso multiusuario.

SELF-e, permitirá a las bibliotecas públicas aceptar presentaciones digitales de autores independientes locales y luego distribuir estos libros electrónicos en las bibliotecas públicas participantes. Y, si un libro electrónico pasa el proceso de selección de Library Journal, se convertirá en parte de un programa de descubrimiento de alcance nacional que permite a los usuarios de la biblioteca poder leer los libros electrónicos seleccionados, y pasarán a formar parte de una plataforma de descubrimiento en todas las bibliotecas participantes en el proyecto. Por el momento hay cinco participantes beta en el programa SELF-e, Biblioteca del Estado de Arizona, Biblioteca Pública de Los Ángeles, Biblioteca Pública del Condado de San Diego, Biblioteca Pública del Condado de 
Cuyahoga, en Ohio, y las Bibliotecas del Estado de Massachusetts.

SELF-e, es una de las novedades más interesantes de la edición independiente, que va a permitir que los autores de ficción o no ficción desconocidos puedan ampliar su base de lectores. Se trata de una forma sencilla y eficaz para catalogar y dar acceso a libros electrónicos de autores locales y construir una comunidad en torno a la escritura del indie, que permite a los usuarios leer libros electrónicos en un modelo de acceso multiusuario, en cualquier dispositivo, en cualquier momento y desde cualquier lugar.

Con el fin de agilizar y facilitar las negociaciones con los editores para adquirir títulos digitales adicionales, DCL ha creado una carta estándar para producir una declaración de entendimiento común con los editores para la compra y adquisición de contenido digital.

Por lo tanto los desafíos que se plantean a las bibliotecas para los próximos años se centran en tres puntos esenciales:

- Invertir en formatos digitales nativos.

- Repensar los modelos de negocio.

- El fortalecimiento de la alianza entre los editores y productores de contenido y las redes de comunicación.

En España la plataforma CIELO de la Universidad de Salamanca dispone de un convenio con la empresa Traficantes de Sueños para incorporar sus libros electrónicos a la misma. En septiembre de este año ha echado a andar el préstamo digital en bibliotecas públicas españolas con eBiblio de Libranda. eBiblio es un proyecto abierto realizado en cooperación con las comunidades autónomas., que permite ser adaptado, y con la posibilidad de incrementar más contenidos en función de los propios intereses y particularidades de cada una de ellas, que posibilitará el aumento de las colecciones, entre estás colecciones debiera contemplarse la posibilidad de incorporar títulos de autoedición, que es una de las líneas que se está siguiendo en casi todos los países de referencia en este campo. (Merlo Vega 2013) 


\section{Conclusión}

En conclusión la biblioteca en el nuevo contexto está obligada a repensarse en función de toda una serie de parámetros nuevos, inherentes al mundo digital en el que los usuarios cada vez están más inversos, conformando nuevos modos de acceso a la información, nuevos productos y nuevos servicios, en sintonía con una sociedad que está cambiando sus hábitos de consumo de información. Tradicionalmente, los bibliotecarios se enteran de nuevos libros a través de publicaciones comerciales, como Library Journal, PW, y School Library Journal. Pero la mayoría de los títulos autoeditados no son revisados por estas revistas. Aunque algunas bibliotecas trabajan con sus comunidades para publicar libros de los autores locales que integran en sus colecciones, de este modo los autores auto-publicados pueden optar a oportunidades para verse representados por el mercado biblioteca. Los libros auto-publicados necesitan tener éxito en algún nivel o ser examinado por los colaboradores a fin de llegar a la atención de estos programas. El proceso de ser reconocido por el mercado de la biblioteca no es necesariamente diferente de conseguir la tracción necesaria a través de editoriales con los lectores y minoristas. Las bibliotecas están seleccionando libros de autores que no han tenido mediación de una editorial para sus colecciones. El que un libro de un autor independiente sea añadido al catálogo de una biblioteca es sólo el primer paso, ya que las bibliotecas ayudan a que los libros sean descubiertos a través del propio catálogo, con pantallas, exposiciones, boletines informativos, eventos o entrevistas con los autores.

Las bibliotecas han empezado a reconocer este hecho y están empezando a hacer cambios en sus procedimientos de compra y presupuestos, para poder disponer de más libros electrónicos autopublicados con el objetivo de reflejar mejor las realidades del público lector. Los autores que deciden publicar sin la mediación de una editorial están produciendo libros relevantes que vale la pena leer, libros que tienen un importante impacto en nuestra cultura, en los individuos, y en las editoriales. Algunos de los best seller más importantes de los últimos años nacieron en los sistemas de autopublicación como es el caso de 50 sombras de Grey, Sangre Letal de Jhon Locke, Ascend e Amanda Hocking o Canciones para Laura del periodista sevillano Blue Jean.

Este es un cambio significativo y tiene consecuencias sobre los servicios de selección y lectura de las bibliotecas. Los bibliotecarios en el pasado no se molestaron mucho en considerar la inclusión de libros autopublicados porque muchos de esos títulos eran de baja calidad o volúmenes de la llamada peyorativamente "Prensa de las vanidades". Sin embargo ahora, los títulos de libros electrónicos de autopublicación se están convirtiendo en una parte importante de nuestra literatura contemporánea y tienen que estar en las 
bibliotecas. Si las bibliotecas de hoy en día quieren cumplir con sus misiones de garantizar la conservación y transmisión a la sociedad del conocimiento, la historia y la cultura, entonces deben tener en cuenta la adquisición de libros electrónicos autopublicados para ponerlos a disposición de sus lectores. Con varios libros autopublicados en lo más alto de las listas de éxitos, y cuando algunos de los escritores más destacados que rompen los lazos con sus editoriales, como es el caso de J. K. Rowling que ha creado Pottermore, el único lugar donde pueden comprarse los ebooks de la serie Harry Potter, no podemos seguir considerando a los libros de autopublicados como elementos de periféricos de la cultura. Las bibliotecas estadounidenses están considerando este asunto y están empezando a cambiar sus hábitos de compra. El trabajo de una biblioteca es aprovechar la inversión cívica y ser un agente intermediario de cooperación para las adquisiciones de los registros más significativos de nuestra cultura. Si las bibliotecas de hoy en día quieren cumplir con sus misiones de "garantizar la conservación y transmisión de la sociedad del conocimiento, la historia y la cultura” (Declaración de la misión de la Biblioteca Pública de Brooklyn, en Nueva York), entonces deben tener en cuenta la adquisición de libros electrónicos autopublicados para ponerlos a disposición de sus lectores. Después de todo, muchos de estos son los más vendidos y son cada vez un mayor porcentaje de la producción total de nuestra cultura.

\section{Bibliografía}

ALONSO-ARÉVALO, J., CORDÓN-GARCÍA, J.A. y GÓMEZ DÍAZ, R. 2014. eBooks en bibliotecas: gestión, tratamiento y aplicaciones. Buenos Aires: Alfagrama. Biblioteca Alfagrama.

CALIFA LIBRARY GROUP 2015a. Disponible en: http://califa.org/.

CONSORTIUM, C.L. 2013. Colorado's eBook Manifesto. [en línea], Disponible en: http://evoke.cvlsites.org/files/2012/07/ColoradoeBookManifesto.pdf.

CORROTO, P. 2015. Las bibliotecas públicas transforman el préstamo en EE UU. eldiario.es [en línea], Disponible en: http://www.eldiario.es/turing/industria_editorial/ bibliotecas-publicas-transforman-prestamo-EEUU_o_345766196.html.

Delivering highly effective eLearning courses. Edtion ed.: Talem ims, 2015b. Disponible en: http://www.talentlms.com/ebook/effective-courses/effectivecourses-jan2015v1.1.pdf.

Enki Library, 2015c. Disponible en:https://enkilibrary.org/.

Evoke! [en línea], 2013d. Disponible en: http://evoke.cvlsites.org/. 
How public libraries are evolving: to meet patrons' needs in the digital age. Overdrive, 2015d, v 10/15. Disponible en: http://blogs.overdrive.com/wp-content/ uploads/2015/10/B2C_EM_9.30.pdf.

KAHLE, B. M., 1993. Interfaces for distributed systems of information servers. Journal of the American Society for Information Science, 44(8). Disponible en: http:// www3.interscience.wiley.com/cgi-bin/jtoc/27981/.

LARA, K. 2014. Open Access Library Survey: An investigation of the role of libraries in open access funding and support within institutions [en línea]. pcgplus.com. Disponible en: http://www.pcgplus.com/wp-content/uploads/2014/o9/PCG-Open-Access-Library -Survey-2014.pdf.

LARUE, J., 2013. Wanna Write a Good One? Library as Publisher: Envisioning a new model outside the Big Six. ALA E-Content Digital Supplement. Disponible en: http:// www.americanlibrariesmagazine.org/article/wanna-write-good-one-library-publisher.

LIBRARIES, D. C., 2013. List of Publishers Willing to Sell to Libraries [en línea]. Disponible en: http://evoke.cvlsites.org/resources-guides-and-more/publishers-willingto-sell-e-content/

LIBRARY-JOURNAL 2014. Ebook Usage in U.S. Public Libraries. Edtion ed. Library Journal. Disponible en: http://www.thedigitalshift.com/research/ebook-usage-u-spublic-libraries-2014-report/.

MERLO VEGA, J.A. 2013. El préstamo de libros electrónicos en las bibliotecas de la Universidad de Salamanca. Trama \& Texturas [en línea], Disponible en: http:// gredos.usal.es/jspui/handle/10366/122485.

POLANKA, S., 2012. No Shelf Required 2: Use and Management of Electronic Books. New York: ALA Editions. ISBN 978-0-8389-1054-2.

Self-e Library Journal, 2015e. Disponible en: http://self-e.libraryjournal.com/.

\section{Notas}

1. Bibliotecario de la Facultad de Traducción y Documentación. Universidad de Salamanca.E-mail: alar@usal.es

2. Bibliotecario de la Biblioteca "Abrahan Zacut". Universidad de Salamanca. Email: rulinha@usal.es 
MEI, II, Vol. 6, no 11, pág. 214 